\title{
Pro-inflammatory Cytokines and Oxidized Low-Density-Lipoprotein in Patients With Fibromyalgia
}

\author{
Funda Canpolat KUTU (iD ${ }^{1}$, Şenay ÖZDOLAP (iD ${ }^{2}$, Selda SARIKAYA(D) ${ }^{2}$ \\ ${ }^{1}$ Department of Physical Medicine and Rehabilitation, Çaycuma State Hospital, Zonguldak, Turkey \\ ${ }^{2}$ Department of Physical Medicine and Rehabilitation, Medicine Faculty of Bülent Ecevit University, Zonguldak, Turkey
}

\begin{abstract}
Objectives: This study aims to evaluate the differences in serum levels of tumor necrosis factor-alpha (TNF-a), interleukin-1 beta (IL-1 $\beta$ ), (IL-8), and oxidized low-density lipoprotein (Ox-LDL) between female fibromyalgia (FM) patients and healthy females and also to examine a possible relationship between the cytokines/biomarker and the severity of pain and clinical features of FM.

Patients and methods: This case-control study included 48 female patients (mean age $26.51 \pm 6.98$ years; range, 18 to 50 years) who were diagnosed with FM according to the American College of Rheumatology criteria and 43 healthy female subjects (mean age $23.93 \pm 4.22$ years; range, 18 to 50 years). Serum levels of TNF-a, IL-1 $\beta$, IL-8, and Ox-LDL were measured by enzyme-linked immunosorbent assay in both groups. Fibromyalgia Impact Questionnaire, visual analog scale, symptom severity scale, and widespread pain index were carried out and also their relationships with TNF- $a, I L-1 \beta, I L-8$, and Ox-LDL levels were evaluated.

Results: There was no difference in levels of the serum TNF- $a, I L-1 \beta, I L-8$, and Ox-LDL between FM patients and healthy controls ( $p>0.05$ ). The severity of pain was significantly associated with TNF- $a(r=0.338 ; p=0.021)$ and IL-8 $(r=0.299 ; p=0.043)$ levels, and Ox-LDL ( $r=0.415 ; p=0.006)$ level was found to be related to symptom severity of FM.

Conclusion: Our results suggest that serum levels of TNF- $a$ and IL-8 may be involved in the occurrence of pain in FM. The level of Ox-LDL may play an important role in the severity of symptoms. Future studies are needed to determine whether and how Ox-LDL plays a role in FM.

Keywords: Cytokines, fibromyalgia, oxidative stress, oxidized-low-density lipoprotein, pain.
\end{abstract}

Fibromyalgia (FM) is a common musculoskeletal disorder characterized by chronic widespread pain, joint stiffness, multiple tender points, and systemic symptoms (i.e., cognitive dysfunction, fatigue, sleep disturbances, and mood disorders) without an underlying organic disease. ${ }^{1}$ It affects nearly $5 \%$ of the overall population worldwide, indicating that it is one of the most common rheumatic diseases, and females are nine-times more susceptible to FM than males. ${ }^{2}$

The pathophysiology of FM has not been completely understood and there are no established etiological factors. The fundamental pathophysiological mechanism of FM has not yet thoroughly been explained even though many studies have been conducted. It has been suggested that FM symptoms originate from the interactions among autonomic nervous system, hypothalamus-pituitary-adrenal (HPA) axis, and immune system. ${ }^{3}$

Although FM is accepted to be a noninflammatory disease, it has been considered that cytokines play a role in its etiology since an earlier study by Wallace in $1988 .{ }^{4}$ An imbalance of pro-

Received: November 13, 2017 Accepted: May 05, 2018 Published online: August 16, 2018

Correspondence: Şenay Özdolap, MD. Çaycuma Devlet Hastanesi Fiziksel Tıp ve Rehabilitasyon Kliniği, 67900 Çaycuma, Zonguldak, Turkey. Tel: +90 372 - 2612463 e-mail: senayozdolap@hotmail.com 
inflammatory and anti-inflammatory cytokines has been proposed to be involved in pain induction and maintenance. However, several studies have shown contradictory results in cytokine levels in this patient population. ${ }^{5}$ Besides, it has been shown that cytokines are engaged with the communication between the immune and nervous systems. ${ }^{6}$ In addition, several studies have suggested that there is a connection between several cytokines (tumor necrosis factor-alpha $[$ TNF- $\alpha$ ], interleukin-1 beta [IL-1 $]$ ], IL-6, IL-8) and some of the symptoms of FM including hyperalgesia, fatigue, sleep disorders, cognitive dysfunctions, anxiety, and stress. ${ }^{7-9}$ However, how cytokines affect the functions of the central nervous system and what their roles are in creating widespread pain in FM are still the questions to be answered.

In recent studies, some evidences have suggested that oxidative stress may also have a role in the pathophysiology of FM..$^{2,10}$ In patients with FM, oxidative stress biomarkers have been significantly found to be elevated. ${ }^{11,12}$ Oxidized low-density lipoprotein (Ox-LDL) is one of the markers of oxidative stress which identifies an association between the immune system and abnormal inflammation. ${ }^{13}$ Pro-inflammatory effect is one of the features of this lipoprotein which also increases inflammatory cytokine production in addition to cell adhesion, migration, proliferation, and apoptosis. ${ }^{13,14}$ Circulating Ox-LDL increases in certain diseases which are associated with high oxidative stress and inflammation. ${ }^{15}$ These results have led us to investigate the expression of cytokines, as well as Ox-LDL in FM. Therefore, in this study, we aimed to evaluate the differences in serum levels of TNF- $\alpha$, IL- $1 \beta$, IL- 8 , and Ox-LDL between female FM patients and healthy females and also to examine a possible relationship between the cytokines/biomarker and the severity of pain and clinical features of FM.

\section{PATIENTS AND METHODS}

A total of 318 female patients aged between 18 and 50 years who were diagnosed with FM based on the American College of Rheumatology (ACR) 2010 criteria $^{16}$ in the Zonguldak Bülent Ecevit University, Medicine Faculty, Department of Physical Medicine and Rehabilitation between May 2013 and May 2014 were screened and 48 (mean age 26.51 \pm 6.98 years; range, 18 to 50 years) were included in this study according to ex-in-criteria. The control group consisted of 43 healthy female hospital workers (mean age $23.93 \pm 4.22$ years; range, 18 to 50 years) who did not have any systemic disease.

Inclusion criteria for both groups were as follows: (i) patients aged between 18 and 50, (ii) having no systemic disease other than FM, (iii) being diagnosed with FM for the first time, (iv) being treatment-naive for FM, and (v) having no medication within the last three weeks for any reason. Exclusion criteria were the presence of any inflammatory autoimmune disease, cardiovascular, neurologic, renal, metabolic and endocrine problems, pregnancy or breast-feeding, and a diagnosis of FM in the control subjects.

Demographic characteristics of both FM patients and healthy controls were recorded and the body mass index (BMI) was calculated for each individual $\left(\mathrm{kg} / \mathrm{m}^{2}\right)$. The pain severity was assessed using the visual analog scale (VAS)-pain scores $(0-10 \mathrm{~cm})$. The numbers of tender points were counted according to the digital palpation which is performed by pressing the tender areas with approximately $4 \mathrm{~kg} / \mathrm{cm}^{2}$ pressure, until the pulp of the thumb whitened. For a tender point to be positive, the subject must state that the palpation is painful. Tender points count (TPC) was carried out according to the ACR 1990 FM diagnosis criteria. ${ }^{17}$ The Widespread Pain Index (WPI) and Symptom Severity Scale (SSS) scores of each patient were calculated according to the ACR 2010 FM diagnosis criteria. ${ }^{16}$ In addition, functional status and disease severity of the patient group were evaluated using the Fibromyalgia Impact Questionnaire (FIQ). ${ }^{18}$ This instrument was found to be reliable and valid in Turkish female FMS patients. ${ }^{19}$

Blood samples of the patient and control groups were drawn after an overnight fasting. The levels of TNF- $\alpha$, IL-1 $\beta$, IL- 8 ELISA assays (Platinum ELISA; eBioscience, San Diego, California, USA), and Ox-LDL (Biomedica, Baster, Vienna, Austria) were measured using the enzyme-linked immunosorbent assay (ELISA) according to the manufacturer's instructions. 


\begin{tabular}{|c|c|c|c|}
\hline \multirow[t]{2}{*}{ Demographic data and clinical variables } & FM patients $(n=48)$ & Healthy controls $(n=43)$ & \\
\hline & Mean \pm SD & Mean \pm SD & $p$ \\
\hline Age (year) & $26.5 \pm 7.0$ & $23.9 \pm 4.2$ & 0.159 \\
\hline Body mass index $\left(\mathrm{kg} / \mathrm{m}^{2}\right)$ & $21.7 \pm 3.3$ & $21.8 \pm 3.4$ & 0.993 \\
\hline Visual analog scale score & $5.7 \pm 2.0$ & $1.3 \pm 1.9$ & $<0.001$ \\
\hline Symptom severity scale score & $7.1 \pm 1.9$ & $1.4 \pm 1.6$ & $<0.001$ \\
\hline Widespread pain index score & $9.9 \pm 3.1$ & $1.0 \pm 1.3$ & $<0.001$ \\
\hline Tender points count & $13.9 \pm 2.3$ & $0.9 \pm 1.2$ & $<0.001$ \\
\hline Fibromyalgia impact questionnaire score & $50.3 \pm 14.5$ & $22.0 \pm 13.9$ & $<0.001$ \\
\hline
\end{tabular}

A written informed consent was obtained from each participant. The study was approved by the Ethics Committee of Bulent Ecevit University (2013-32-26/02), Zonguldak, Turkey, and was conducted in accordance with the principles of the Declaration of Helsinki.

\section{Statistical analysis}

Statistical analysis was performed using the IBM SPSS version 19.0 software (IBM Corp., Armonk, NY, USA). Continuous variables were expressed in mean \pm standard deviation or median (min-max) values, while categorical variables were expressed in number and percentage. Normality of the distribution of the data was analyzed using the Shapiro-Wilk test. Continuous variables were compared using the independent sample t-test or Mann-Whitney U test, while categorical variables were compared using the Yates corrected chisquare test or Fisher's exact chi-square test. The Spearman's correlation analysis was performed to examine the relationship between the continuous variables. Linear regression was performed for multivariate analysis. $\mathrm{P}$ values of less than 0.05 were considered statistically significant.

\section{RESULTS}

A total of $48 \mathrm{FM}$ patients and 43 healthy individuals were included in this study. The mean age was $26.51 \pm 6.98$ years and $23.93 \pm 4.22$ years in the patient and control groups, respectively, indicating no statistically significant difference between the groups $(p=0.159)$.

The mean BMI was $21.65 \pm 3.32 \mathrm{~kg} / \mathrm{m}^{2}$ in the patient group and $21.80 \pm 3.38 \mathrm{~kg} / \mathrm{m}^{2}$ in the control group, indicating no statistically significant difference between the two groups $(p=0.993)$. Demographic characteristics were similar between the groups (Table 1). However, the WPI, SSS, VAS, FIQ, and TPC scores were statistically significantly higher in the FM group compared to the control group $(\mathrm{p}<0.001)$ (Table 1). According to linear regression analysis, WPI and TPC were significant predictors of SSS.

Table 2. Serum tumor necrosis factor-alpha, interleukin-1 beta, interleukin-8, and oxidized low-density lipoprotein levels in FM patients and healthy controls

\begin{tabular}{lcccc}
\hline & FM patients $(\mathrm{n}=48)$ & & Healthy controls $(\mathrm{n}=43)$ & \\
\cline { 2 - 2 } & Mean \pm SD & & Mean \pm SD & $p$ \\
\hline Tumor necrosis factor alpha & $75.0 \pm 59.2$ & & $95.5 \pm 57.2$ & 0.144 \\
Interleukin-1 $\beta$ & $26.8 \pm 21.7$ & & $34.4 \pm 27.4$ & 0.284 \\
Interleukin-8 & $231.2 \pm 249.4$ & & $136.0 \pm 201.0$ & 0.106 \\
Oxidized low density lipoprotein & $11.7 \pm 16.6$ & & $6.8 \pm 5.1$ & 0.379 \\
\hline FM: Fibromyalgia; SD: Standard deviation. & & & \\
\hline
\end{tabular}




\begin{tabular}{|c|c|c|c|c|}
\hline Clinical variables & TNF- $\alpha$ & IL-1 $\beta$ & IL-8 & Ox-LDL \\
\hline Visual analog scale score & $0.338^{*}$ & 0.192 & $0.299^{*}$ & 0.221 \\
\hline Symptom severity scale score & 0.138 & 0.191 & -0.005 & $0.415^{*}$ \\
\hline Widespread pain index score & 0.085 & 0.099 & 0.007 & -0.020 \\
\hline Tender points count & -0.079 & 0.111 & -0.161 & 0.175 \\
\hline Fibromyalgia impact questionnaire score & 0.274 & 0.024 & 0.279 & 0.049 \\
\hline
\end{tabular}

In this study, there was no statistically significant difference in the levels of serum pro-inflammatory cytokines (TNF- $\alpha$, IL-1 $\beta$, IL- 8 ) and Ox-LDL between the patient and control groups ( $p>0.05$ ) (Table 2). However, there was a significant positive correlation between the VAS scores and serum IL- 8 and TNF- $\alpha$ levels $(r=0.299$, $\mathrm{p}=0.043 ; \mathrm{r}=0.338, \mathrm{p}=0.021$, respectively) in the FM group. Although the strength was weak, a significant positive correlation was found between the Ox-LDL levels and SSS scores of the patients in the FM group $(\mathrm{r}=0.415, \mathrm{p}=0.006)$ (Table 3). Correlations between serum cytokines and clinical variables in the FM patients are shown in Table 3.

\section{DISCUSSION}

In this study, the levels of serum pro-inflammatory cytokines (TNF- $\alpha$, IL-1 $\beta$, IL-8) and oxidative stress biomarker (Ox-LDL) were investigated in patients with FM and compared with the control group. The levels of serum pro-inflammatory cytokines and Ox-LDL in the patient group were similar to the healthy individuals. The levels of TNF- $\alpha$ and IL-8 were related to pain severity. In addition,
Ox-LDL levels of the patients were related to severity of symptoms.

In the literature, controversial results in several studies investigating serum pro-inflammatory cytokine levels in patients with FM have been reported. Some authors have reported normal TNF- $\alpha$, IL-1 $\beta$, and IL- 8 levels, while others have shown high levels of those cytokines.,20-22 In addition, new opinions during recent years have claimed that cytokines may play a role in the pathogenesis and be responsible for many symptoms of FM.5,8 Table 4 summarizes the relationship between certain cytokines and the FM symptoms. ${ }^{5,22}$ It has been reported that cytokines such as TNF- $\alpha$, IL-1, IL- 6 , and IL- 8 contribute to the development of peripheral neuropathy, they are associated with HPA and sympathetic nervous system, and catecholamines ensure the release of these cytokines. ${ }^{8,23}$

The IL- 8 causes hyperalgesia, activating the sympathetic nervous system by means of a mechanism independent from prostaglandins. ${ }^{24,25}$ In addition, it has been shown that glial cell activation in chronic pain, such as FM, results in central sensitization and hyperalgesia. ${ }^{24,25}$

Table 4. Cytokines and their associations with symptoms potentially relevant to fibromyalgia

\begin{tabular}{ll}
\hline Cytokines & Association \\
\hline TNF- $\alpha$ & Stress, REM sleep, allodynia, fatigue, depression \\
IL-1 $\beta$ & Stress, fever, fatigue, sleep, myalgia \\
IL-2 & Myalgia, cognitive dysfunction \\
IL-6 & Stress, fatigue, hyperalgesia, depression, activates sympathetic nervous system \\
IL-8 & Substance P stimulates production, sympathetic pain, REM sleep \\
IL-10 & Blocks pain \\
\hline
\end{tabular}

TNF- $\alpha$ : Tumor necrosis factor-alpha; REM: Rapid eye movement; IL-1 $\beta$ : Interleukin-1 beta; IL-2: Interleukin-2; IL-6: Interleukin-6; IL-8: Interleukin-8; IL-10: Interleukin-10. 
Glial cell activation causes the release of cytokines such as TNF- $\alpha$, IL-1 $\beta$, and IL- 8 . There are several studies indicating that the level of IL-8 in FM is not different from that of healthy individuals, ${ }^{9,26}$ while some others have found higher levels in FM patients.,21 Gur et al. ${ }^{27}$ reported that IL-8 was related to pain severity in patients with FM. Kadetoff et al. ${ }^{25}$ also found higher IL- 8 levels in the cerebrospinal fluid of 15 patients with FM, compared to the control group, and concluded that IL-8 was responsible for hyperalgesia. The correlation between the FIQ and IL-8 has also been shown in a very recent study. ${ }^{21}$ In our study, although the level of IL-8 in the fibromyalgia group was not significantly different compared to the control group, a significant correlation between the VAS scores and IL-8 levels of the patients was found suggesting that IL- 8 can affect the pain severity. We consider that the correlation between the VAS and IL- 8 supports that IL- 8 may lead to hyperalgesia.

Furthermore, many endogenous and exogenous impacts of IL- 1 such as weight loss, sleep regulation, regulation of the immune system and nervous system functions, neuronal transmission, epilepsy, and nerve cell death have been demonstrated. ${ }^{28}$ IL-1 is produced by the liver and induces hyperalgesia with vagal stimuli. It also regulates the release of substance $P$, thereby, contributing to the development of fever. ${ }^{28}$ Although it is considered that IL-1 contributes to symptoms such as somnolence, hyperalgesia, fatigue, myalgia, and depression in patients with $\mathrm{FM},{ }^{22}$ many studies have shown that IL- $1 \beta$ levels are similar among patients with FM and healthy individuals. ${ }^{20,22,27}$ In one report, IL-1 levels were lower in 64 patients with FM. ${ }^{29}$ Accordingly, we detected no statistically significant difference between patients with FM and healthy controls in terms of the IL- $1 \beta$ levels, and therefore the IL- $1 \beta$ levels did not correlate with any parameters of disease severity.

Tumor necrosis factor-alpha is produced when the organism is exposed to any inflammatory or infectious stimuli. The major biological role of TNF- $\alpha$ is to defend the organism against bacterial, viral, or parasitic infections. ${ }^{30}$ In addition, TNF- $\alpha$ synthesis occurs by means of stress, substance $\mathrm{P}$ release, rapid eye movement (REM) sleep, allodynia, norepinephrine, and epinephrine stimulation. ${ }^{8}$ It has been proposed that TNF- $\alpha$ is responsible for hyperalgesia, myalgia, fatigue, depression, and REM sleep in FM patients. ${ }^{8}$ In our study, there was no significant difference in the serum TNF- $\alpha$ levels between the patient and control groups. However, we found a statistically significant correlation between the VAS scores and serum TNF- $\alpha$ levels in the FM patients. This finding supports the hypothesis that the TNF- $\alpha$ levels can affect the severity of pain, thereby, resulting in hyperalgesia in patients with FM.

To the best of our knowledge, this is the first study investigating the correlation between Ox-LDL and FM. The former is an indicator of oxidative stress, which ensures correlation between inflammation and immune system. ${ }^{14}$ It has pro-inflammatory effects and increases the release of inflammatory cytokines and cell adhesion, migration, proliferation, and apoptosis. Oxidized low-density lipoprotein occurs in many diseases due to oxidative stress, such as atherosclerosis, sepsis, and chronic obstructive pulmonary disease. ${ }^{15,31} \mathrm{In}$ recent years, several studies have been conducted examining the oxidative system in FM patients. These studies have suggested that oxidative stress contributes to the pathophysiology of the disease and have shown lower antioxidant activity in FM population. ${ }^{2,10}$ Low antioxidant levels are unable to compete with the reactive oxygen species (ROS). This causes the accumulation of ROS inside the cells and then the lipid peroxidation and in turn, destruction of polyunsaturated fatty acids of the cell membrane, resulting in cell death. ${ }^{2}$ In patients with FM, there is a potential connection between the inflammatory process and oxidative stress. In light of these data, we evaluated serum levels of Ox-LDL in the FM patients. We found no significant difference in the Ox-LDL levels between the FM patients and healthy controls. Interestingly, however, we found a positive correlation between the Ox-LDL levels and SSS scores of the FM patients. In the literature, there is no such correlation reported earlier, although there are several studies showing the correlation between lipid peroxidation and pain, depression, and FIQ scores. ${ }^{32,33}$ Although we detected similar serum Ox-LDL levels in the FM patients and healthy controls, we cannot conclude that Ox-LDL certainly does not play a role in the etiopathogenesis of FM. Therefore, further, largescale studies are still needed to investigate the levels of serum Ox-LDL in patients with FM. 
The contradictory results of previous studies on the role of pro-inflammatory cytokines in the etiopathogenesis of FM might have resulted from different parameters used such as the inclusion criteria, presence of comorbidities, use of medications, symptom patterns and duration of FM, age, and BMI of the patients, and physical activity patterns. In the present study, we attempted to avoid the factors which might affect the levels of serum cytokines as far as possible, by excluding patients with comorbidities. We also avoided the potential role of obesity in inflammation since the mean BMI was very similar and normal in both groups. These are strengths of our study. However, the small sample size is the main limitation of our study, necessitating further large-scale studies.

In conclusion, our results do not support that the levels of cytokines (TNF- $\alpha$, IL-I $\beta$, IL- 8 ) and biomarker of the oxidative stress (Ox-LDL) are elevated in FM patients, although the results suggest that pain experienced by FM patients may be correlated with the levels of TNF- $\alpha$, IL- 8 , and Ox-LDL. However, well-designed, large-scale studies are required to confirm these findings.

\section{Acknowledgement}

The authors would like to thank Dr. İshak Özel Tekin MD for his precious dedication and care in the studies of tumor necrosis factor-alpha-blocker and oxidized lowdensity lipoprotein and to Çağatay Büyükuysal, Ph.D. and Füruzan Köktürk, Ph.D. for their contributions in the statistical analyses of the data.

\section{Declaration of conflicting interests}

The authors declared no conflicts of interest with respect to the authorship and/or publication of this article.

\section{Funding}

This study was supported by Bulent Ecevit University Scientific Research Fund (Project Number: 201393733444-01).

\section{REFERENCES}

1. Mease P. Fibromyalgia syndrome: review of clinical presentation, pathogenesis, outcome measures, and treatment. Rheumatol Suppl 2005;75:6-21.

2. Pernambuco AP, Leite Schetino LP, Cota Carvalho LDS, Reis DD. Involvement of oxidative stress and nitric oxide in fibromyalgia. Fibrom Open Access 2016;1:1000105.
3. Ang DC, Moore MN, Hilligoss J, Tabbey R.MCP-1 and IL-8 as pain biomarkers in fibromyalgia: a pilot study. Pain Med 2011;12:1154-61.

4. Wallace DJ, Margolin K, Waller P.Fibromyalgia and interleukin-2 therapy for malignancy. Ann Intern Med 1988;108:909.

5. Uçeyler N, Häuser W, Sommer C. Systematic review with meta-analysis: cytokines in fibromyalgia syndrome. BMC Musculoskelet Disord 2011;12:245.

6. Staud R.Cytokine and immune system abnormalities in fibromyalgia and other central sensitivity syndromes. Curr Rheumatol Rev 2015;11:109-15.

7. Bazzichi L, Rossi A, Massimetti G, Giannaccini G, Giuliano T, De Feo F, et al. Cytokine patterns in fibromyalgia and their correlation with clinical manifestations. Clin Exp Rheumatol 2007;25:225-30.

8. Rodriguez-Pintó I, Agmon-Levin N, Howard A, Shoenfeld Y. Fibromyalgia and cytokines. Immunol Lett 2014;161:200-3.

9. Xiao Y, Haynes WL, Michalek JE, Russell IJ. Elevated serum high-sensitivity $C$ reactive protein levels in fibromyalgia syndrome patients correlate with body mass index, interleukin-6, interleukin-8, erythrocyte sedimentation rate. Rheumatol Int 2013;33:1259-64.

10. Ranzolin A, Duarte AL, Bredemeier M, da Costa Neto CA, Ascoli BM, Wollenhaupt-Aguiar B, et al. Evaluation of cytokines, oxidative stress markers and brain-derived neurotrophic factor in patients with fibromyalgia - A controlled cross-sectional study. Cytokine 2016;84:25-8.

11. Chung CP, Titova D, Oeser A, Randels M, Avalos I, Milne GL, et al. Oxidative stress in fibromyalgia and its relationship to symptoms. Clin Rheumatol 2009;28:435-8.

12. Ozgocmen S Ozyurt H, Sogut S, Akyol O, Ardicoglu O, Yildizhan H. Antioxidant status, lipid peroxidation and nitric oxide in fibromyalgia: etiologic and therapeutic concerns. Rheumatol Int 2006;26:598-603.

13. Tekin IO, Orem A, Shiri-Sverdlov R. Oxidized LDL in inflammation: from bench to bedside. Mediators Inflamm 2013;2013:762759.

14. Greig FH, Kennedy S, Spickett CM. Physiological effects of oxidized phospholipids and their cellular signaling mechanisms in inflammation. Free Radic Biol Med 2012;52:266-80.

15. Shen Y, Yang T, Guo S, Li X, Chen L, Wang T, et al. Increased serum ox-LDL levels correlated with lung function, inflammation, and oxidative stress in COPD. Mediators Inflamm 2013;2013:972347.

16. Wolfe F, Clauw DJ, Fitzcharles MA, Goldenberg DL, Katz RS, Mease P, et al. The American College of Rheumatology preliminary diagnostic criteria for fibromyalgia and measurement of symptom severity. Arthritis Care Res (Hoboken) 2010;62:600-10.

17. Wolfe F, Smythe HA, Yunus MB, Bennett RM, Bombardier C, Goldenberg DL, et al. The American College of Rheumatology 1990 Criteria for the 
Classification of Fibromyalgia. Report of the Multicenter Criteria Committee. Arthritis Rheum 1990;33:160-72.

18. Burckhardt CS, Clark SR, Bennett RM. The fibromyalgia impact questionnaire: development and validation. J Rheumatol 1991;18:728-33.

19. Sarmer S, Ergin S, Yavuzer G. The validity and reliability of the Turkish version of the Fibromyalgia Impact Questionnaire. Rheumatol Int 2000;20:9-12.

20. Wang H, Moser M, Schiltenwolf M, Buchner M. Circulating cytokine levels compared to pain in patients with fibromyalgia -- a prospective longitudinal study over 6 months. J Rheumatol 2008;35:1366-70.

21. Mendieta D, De la Cruz-Aguilera DL, BarreraVillalpando MI, Becerril-Villanueva E, Arreola R, Hernández-Ferreira E, et al. IL-8 and IL-6 primarily mediate the inflammatory response in fibromyalgia patients. J Neuroimmunol 2016;290:22-5.

22. Wallace DJ, Linker-Israeli M, Hallegua D, Silverman S, Silver D, Weisman MH.Cytokines play an aetiopathogenetic role in fibromyalgia: a hypothesis and pilot study. Rheumatology (Oxford) 2001;40:743-9.

23. Malek H, Ebadzadeh MM, Safabakhsh R, Razavi A, Zaringhalam J. Dynamics of the HPA axis and inflammatory cytokines: Insights from mathematical modeling. Comput Biol Med 2015;67:1-12.

24. Gur A, Karakoc M, Erdogan S, Nas K, Cevik R, Sarac AJ. Regional cerebral blood flow and cytokines in young females with fibromyalgia. Clin Exp Rheumatol 2002;20:753-60.

25. Kadetoff D, Lampa J, Westman M, Andersson $\mathrm{M}$, Kosek E. Evidence of central inflammation in fibromyalgia-increased cerebrospinal fluid interleukin-8 levels. J Neuroimmunol 2012;242:33-8.

26. Uçeyler N, Valenza R, Stock M, Schedel R, Sprotte G, Sommer C. Reduced levels of antiinflammatory cytokines in patients with chronic widespread pain. Arthritis Rheum 2006;54:2656-64.

27. Gür A, Karakoç M, Nas K, Remzi, Cevik, Denli $\mathrm{A}$, et al. Cytokines and depression in cases with fibromyalgia. J Rheumatol 2002;29:358-61.

28. Dinarello CA. Interleukin-1, interleukin-1 receptors and interleukin-1 receptor antagonist. Int Rev Immunol 1998;16:457-99.

29. Hernandez ME, Becerril E, Perez M, Leff P, Anton B, Estrada S, et al. Proinflammatory cytokine levels in fibromyalgia patients are independent of body mass index. BMC Res Notes 2010;3:156.

30. Bradley JR.TNF-mediated inflammatory disease. J Pathol 2008;214:149-60.

31. Jin W, Zhao Y, Yan W, Cao L, Zhang W, Wang M, et al. Elevated circulating interleukin-27 in patients with coronary artery disease is associated with dendritic cells, oxidized low-density lipoprotein, and severity of coronary artery stenosis. Mediators Inflamm 2012;2012:506283

32. Cordero MD, Alcocer-Gómez E, Cano-García FJ, De Miguel M, Carrión AM, Navas P, et al. Clinical symptoms in fibromyalgia are better associated to lipid peroxidation levels in blood mononuclear cells rather than in plasma.PLoS One 2011;6:26915.

33. La Rubia M, Rus A, Molina F, Del Moral ML. Is fibromyalgia-related oxidative stress implicated in the decline of physical and mental health status? Clin Exp Rheumatol 2013;31:121-7. 